\title{
Measuring the Cognitive Load of Learning to Program: A Replication Study
}

\author{
Albina Zavgorodniaia, Rodrigo Duran, Arto Hellas, Otto Seppälä, and Juha Sorva \\ \{albina.zavgorodniaia,rodrigo.duran, arto.hellas, otto.seppala,juha.sorva\}@aalto.fi \\ Department of Computer Science, Aalto University, Finland
}

\begin{abstract}
Cognitive load (CL) on a learner's working memory has emerged as an influential concept in computing education and beyond. CL is commonly divided in at least two components, intrinsic load (IL) and extraneous load (EL). We seek progress on two questions: (1) How can CL components be measured in the programming domain? (2) How should CL measurement deal with the "third component" of germane load (GL)? We replicate two studies: Morrison and colleagues' [49] evaluation of a questionnaire for self-assessing CL in programming, which is an adaptation of a generic instrument; and Jiang and Kalyuga's [24] study, which found support for a twocomponent measure of CL in language learning, with GL redundant. We crowd-sourced CL data using Morrison's questions at the end of a video tutorial on programming for beginners. A confirmatory factor analysis found strong support for a three-factor model, with factors matching the items intended to capture IL, EL, and GL, respectively. A two-factor model with IL-targeting and GL-targeting items combined gave a poorer fit. Our findings strengthen the claims of discriminant validity and internal reliability for Morrison's CL questionnaire for programming; construct validity for GL remains open, however. We affirm the need for further research on the twocomponent theory of CL and the sensitivity of CL self-assessments to contextual factors.
\end{abstract}

\section{CCS CONCEPTS}

- Social and professional topics $\rightarrow$ Computing education.

\section{KEYWORDS}

cognitive load, programming education, measurement, replication

\section{ACM Reference Format:}

Albina Zavgorodniaia, Rodrigo Duran, Arto Hellas, Otto Seppälä, and Juha Sorva. 2020. Measuring the Cognitive Load of Learning to Program: A Replication Study. In United Kingdom \& Ireland Computing Education Research conference. (UKICER '20), September 3-4, 2020, Glasgow, United Kingdom. ACM, New York, NY, USA, 7 pages. https://doi.org/10.1145/3416465.3416468

\section{INTRODUCTION}

Cognitive load theory (CLT) $[63,65]$ has established itself as one of the leading theoretical frameworks to support instructional design.

Permission to make digital or hard copies of all or part of this work for personal or classroom use is granted without fee provided that copies are not made or distributed for profit or commercial advantage and that copies bear this notice and the full citation on the first page. Copyrights for components of this work owned by others than the author(s) must be honored. Abstracting with credit is permitted. To copy otherwise, or republish, to post on servers or to redistribute to lists, requires prior specific permission and/or a fee. Request permissions from permissions@acm.org.

UKICER '20, September 3-4, 2020, Glasgow, United Kingdom

(C) 2020 Copyright held by the owner/author(s). Publication rights licensed to ACM. ACM ISBN 978-1-4503-8849-8/20/09 ..\$15.00

https://doi.org/10.1145/3416465.3416468
Its goal is to help learners construct new knowledge consciously, as opposed to other educational objectives, such as the activation of learners' prior knowledge or unconscious automation through repeated performance of a familiar task [27]. CLT deals with the learning of domain-specific knowledge that is biologically secondary [63] in that its value is a cultural construct unsupported by biological evolution-programming knowledge is a good example.

CLT highlights how the severe limitations of humans' working memory constrain our ability to learn as we process new information. From this premise, the theory has spawned many specific design principles, or cognitive-load "effects" [63], examples of which include the worked-example effect, which promotes studying examples over problem-solving while prior knowledge is low, and the split-attention effect, which recommends integrating instructional explanations into diagrams rather than presenting them separately.

In computing education research (CER), CLT has been applied to example-based learning $[40,60,70]$, course design $[8,61,66]$, multimedia and visualizations $[46,48,59]$, novel practice tasks [15, 20 , and complexity analysis [14], among other things. One review identified CLT as one of CER's more common theories [37].

One of the major open questions in CLT is how to measure cognitive load; work is also needed on adapting generic cognitive-load measures to the programming domain. We investigate these questions by replicating two studies. We will state our specific research questions in Section 3 after providing some background for them.

\section{RELATED WORK}

\subsection{Basic Concepts of Cognitive Load Theory}

Cognitive load theory rests on the widely accepted model of human cognitive architecture whose central components are working memory and long-term memory [63]. Working memory (WM) is extremely limited: it can hold only a handful of elements at a time, for a short time. This is a bottleneck for learning, as all new information must be first consciously processed in WM. On the other hand, long-term memory is virtually unlimited in capacity and stores domain-specific knowledge organized in schemas. Once learned, even a complex schema can be treated as a single element in WM, which is why people can deal with complex situations in familiar domains. For CLT, learning essentially means schema construction.

Cognitive load (CL) refers to the demands imposed by a learning situation-i.e., materials and activities-on a learner's working memory; this is the intensity of cognitive activity required for a specific learning goal during a narrow time frame [27]. To estimate that intensity, CLT uses the concept of element interactivity, which refers to how many elements the situation requires the learner to simultaneously and consciously hold in WM. Too much element interactivity means cognitive overload and unsuccessful learning.

CLT identifies two types of load: intrinsic load (IL) and extraneous load (EL). The two are cut from the same cloth: they both stem 
from element interactivity and load WM similarly. The difference is analytic: IL is the load that is unavoidable when aiming for a specific learning objective with a certain level of prior knowledge, and EL is the avoidable load that results from suboptimal materials and activities. EL and IL add up to overall CL. [25, 62, 63]

\subsection{Germane Load, Old CLT, and New CLT}

The term "germane load" (GL) is associated with the idea that learning does not happen without WM activity; it refers to this positive aspect of cognitive load. However, the more precise definition and role of GL in CLT has shifted during the past decades. The ripples of that change are still evident in the current literature, which draws on various versions of CLT.

The 1998 formulation of the theory [65], which we will call "Old CLT," defined GL as an independent, comparable source of load alongside IL and EL. In this view, GL is a third, additive component representing load that makes learning happen and should be maximized; IL and EL should be low enough for GL to also "fit in" working memory. Some scholars stressed that GL is not just any useful load but arises from those additional, effortful aspects of learning that go beyond task performance, such as conscious reflection and self-explanation [25, 57]. When interventions based on Old CLT target improvements to motivation and deep engagement with learning materials, they are often phrased as increasing GL.

Old CLT was found to be problematic. Leading figures in CLT complained that it suffered from conceptual flaws, failed to explain various empirical results, used a GL construct that was unnecessary and unjustified by results, and led to unfalsifiable circular interpretations of findings $[25,57,62]$. Some who developed measurement instruments for Old CLT [33] eventually gave up on the GL part of the theory $[5,9,34]$. In 2010, CLT was reformulated.

"New CLT" [25,62] is neater and narrower. The major change is the removal of GL as a third, additive component of CL. All learningeven that resulting from "additional" reflection-results from dealing with IL. New CLT tends to view motivation as something that is relevant to learning but external to the theory; motivated engagement is not an additional load on a learner's mind, but a part of dealing with IL. In New CLT, the term "germane load" has been (perhaps confusingly) repurposed to mean something conceptually quite distinct from IL, EL, and Old CLT's GL. It refers to the actual use of WM resources by a learner to process IL, which happens during learning assuming there is sufficient motivation. Overall, GL tends to play a reduced role in studies that are based on New CLT.

The interpretation of the GL construct is important (to the present work and otherwise) not least because GL is central to debates around empirical measurement of CL, as discussed below.

\subsection{Measuring Cognitive Load}

Methods of CL measurement are commonly grouped into objective and subjective ones. We will briefly comment on objective measures but focus on those subjective measures that relate directly to our work. For broader and deeper reviews, see, e.g., [31, 63, 69].

2.3.1 Objective measures of CL. So-called objective CL measures do not require learners to reflect on and evaluate their mental effort or learning. Many of these measures are based on physiological data sources, such as such as eye-tracking [21], pupillometry [16, 45], heart rate [54], and neuronal [3, 67] and electrodermal [52, 58] activity. Objective measures also include dual-task experiments where some learners are required to perform a secondary task during learning so that cognitive load may be estimated from the differences in resulting performance (e.g. [6]).

Objective methods do not depend on learners' self-assessment abilities. Another benefit is that many of them can be applied "online"i.e., during learning-as an index to real-time changes in load. On the downside, many objective methods are intrusive and distracting. Moreover, most objective measures cannot differentiate between the different types of load, which lofty goal has been suggested as CLT's "holy grail" [4] and "mission impossible" [29]. Differential measurement of loads would enable more detailed predictions and add incisiveness to interpretations, and it might be useful to practitioners as well as researchers. Although some attempts have been made to differentiate loads using objective methods [4], most attempts to date rely on subjective ratings instead.

2.3.2 Subjective measures of CL. Subjective CL measures ask learners to report on their perceived mental effort or other aspects of a learning episode that are expected to indicate cognitive load. Paas's [53] unidimensional nine-point scale for mental effort from 1992 is the time-tested instrument that continues to be used. However, this simple scale cannot differentiate between load types and suffers from noisy measurements [32].

Several groups of researchers have created, adopted, and adapted various questionnaires for subjectively assessing types of CL separately. We will comment on a few.

In 2013, Leppink et al. [33] developed a CL self-assessment questionnaire (quoted in our web appendix [1] for easy reference), which has been since adapted and used by the same group and various others. It asks learners to indicate the level of their agreement with ten claims such as "The activity covered formulas that I perceived as very complex" on an eleven-point scale; IL, EL, and GL are targeted by three, three, and four items, respectively. This initial study took place in a statistics class, but Leppink et al. suggested that the instrument could be readily adapted to other complex knowledge domains by replacing words such as "statistics" and "formula" appropriately. Through factor analysis, Leppink et al. [33] uncovered a three-factor model that fit the data, with factors matching the IL-, EL-, and GL-targeting items, respectively. However, the study failed to support the hypothesis that high scores on the GL-targeting items correlated with better learning, and similar results from a follow-on study [34] led the authors to suggest that the third factor does not represent germane load and to throw their support behind New CLT. In later work, some of the same authors have discarded the name "germane load" in favor of "self-perceived learning" [5] or modified the instrument to focus on IL and EL only [9].

Drawing on Old CLT, Klepsch et al. set out to develop a CL component questionnaire that would be even easier to transfer to different domains than Leppink's. Klepsch et al. also phrased (especially) the GL-targeting items differently, seeking to probe whether students had attempted to understand the lesson completely and holistically (as shown in our web appendix [1]). Much like Leppink et al. [33, 34], Klepsch et al. found that a three-factor model fit their data but the GL-targeting items did not correlate with learning. 
A somewhat different questionnaire was developed by Yeung et al. [68], who identified a four-factor model to predict cognitive load, with factors representing learners' perceptions of difficulty, incompetence, negative affect, and investment of effort.

A recent report by Jiang and Kalyuga [24] sought evidence for New CLT in the language-learning domain. The authors fitted a two-factor model against data collected using a questionnaire similar to that of Leppink et al. [33] (see [1]). They hypothesized that since New CLT's GL represents the allocation of working-memory resources on IL, the IL-targeting and GL-targeting questionnaire items might capture the same construct from different perspectives. A confirmatory factor analysis bore this out: a two-factor model fit the data well, with the EL-targeting items in one factor and the ILand GL-targeting items together in the other. Were this result to be replicated across contexts, it would indicate that the GL-targeting items are redundant and strengthen the case for New CLT.

2.3.3 CL Measurement in Computing Education. There is some research on objective measures of cognitive load in programming. For example, Crk et al. [11,12] used EEG (electroencephalography) during program comprehension, while Andrzejewska and Skawińska [2] tracked eye movements and Nourbakhsh et al. [51] measured galvanic skin response and eye blinks. Duran et al. [14] have proposed CL-inspired methods for analyzing the complexity of computer programs a priori for instructional-design purposes, but the work is so far unsupported by direct empirical evidence.

Subjective mental-effort ratings were used in CER by Mason et al. [42, 43], who surveyed programming students at a coarse level across Australian universities. Once Leppink et al. [33] had published their CL questionnaire for statistics in 2013, Morrison et al. [49] adapted it to programming education (see [1] for the instrument). A factor analysis by Morrison et al. [49] confirmed the questionnaire's internal reliability and discriminant validity in the programming domain: it measured three factors, each of which was internally consistent. Morrison's instrument has been since used in a number of studies in CER (e.g., [15, 20, 38, 48, 50]).

\section{RESEARCH QUESTIONS}

Robins et al. [56] note that Morrison et al.'s [49] CL questionnaire for programming awaits replication. Jiang and Kalyuga [24], having found support for a two-factor CL model in language learning, prompt researchers to "conduct factor analysis on the categories of cognitive load in a broader range of subject domains." The present work responds to these needs. More broadly, we answer recent calls from the CER literature for more replication studies [19] and more work towards standardized instruments of measurement [39].

Our study is opportunistic. We have a recent data set from an as-yet unpublished study, which enables us to replicate analyses from two earlier publications. We ask:

RQ1 Morrison et al. [49] report good discriminant validity and internal reliability for a programming-domain adaptation of Leppink's [33] CL questionnaire, which is based on the threecomponent Old CLT. Do these findings replicate with a different cohort and a different programming tutorial?

RQ2 In a language-learning setting, fiang and Kalyuga [24] used a questionnaire similar to Leppink's [33] and found support for New CLT's two-component model of CL, with GL redundant. Do the findings replicate in the programming domain?
Exploring the robustness of those earlier findings is an incremental step towards solving the broader issues in CL measurement in CER and beyond. Answering these questions will not-and we are not attempting here to-show which version of CLT is "correct." The reader will note that our questions do not directly address construct validity (i.e., whether the instruments actually measure what they are meant to), which is a topic that we will return to in Discussion.

\section{METHODS}

\subsection{Procedure}

Participants were recruited and paid using Amazon MTurk ${ }^{1}$, a platform for crowd-sourcing work that requires human intelligence.

Although already established as a powerful resource for research, crowd-sourcing platforms suffer from variable quality of data [7, 23]. To alleviate this issue, we only accepted participants with 500 approved tasks on MTurk and a task-approval rate of at least $98 \%$.

Participation consisted of three stages: (1) a demographic survey, (2) an instructional video on programming, and (3) a postinstructional survey. The demographic survey asked about the participants' previous programming experience among various other background questions and was designed to avoid hinting at the subsequent programming-related instruction. The instructional video is described below. The post-instructional survey contained a cognitiveload questionnaire (see below) and a post-test on programming that is outside the scope of this article.

4.1.1 Instructional Video. The 24-minute instructional video was a beginner-level lesson to reading Python code, in English. The video introduced the concepts of variable, expression, and value and taught the participants to reason about short fragments of imperative code consisting of assignment statements and print commands. The video covered several short example programs, explaining the code constructs and tracing each programs' behavior in detail. The video was configured so that once the participant clicked Play, it could not be paused, rewound, or forwarded.

Because we collected this data in the context of an experiment on presentation modalities in instructional video (not reported here), the participants were randomly given one of three variants of the same video. The variants differed in whether the program examples were accompanied by text, audio, or both. As we observed no significant differences in CL scores between these groups, we are combining the groups for the analysis presented herein.

4.1.2 Cognitive-Load Questionnaire. To measure cognitive load, we used the instrument previously adapted for programming by Morrison et al. [49] from Leppink et al.'s [33] original. Shown in Figure 1, Items 1 to 3 target IL; items 4 to $6 \mathrm{EL}$, and items 7 to $10 \mathrm{GL}$. Below, we will refer to the items as I1-I3, E1-E3, and G1-G4, respectively. A minor difference between our instrument and Morrison's is that we used a ten-point scale while they used an eleven-point one.

\subsection{Participants}

A total of 307 MTurk workers participated. For the analysis presented here, we excluded 132 responses, leaving us with 175 . Sixty responses were excluded due to prior knowledge: we only included

\footnotetext{
${ }^{1}$ https://www.mturk.com/
} 
All of the following questions refer to the lecture that just finished. Please respond to each of the questions on the following scale by circling the appropriate number. (1 meaning not at all the case, and 10 meaning completely the case)

(1) The topics covered in the activity were very complex.

(2) The activity covered program code that I perceived as very complex.

(3) The activity covered concepts and definitions that I perceived as very complex.

(4) The instructions and/or explanations during the activity were very unclear.

(5) The instructions and/or explanations were, in terms of learning, very ineffective.

(6) The instructions and/or explanations were full of unclear language.

(7) The activity really enhanced my understanding of the topic(s) covered.

(8) The activity really enhanced my knowledge and understanding of computing/programming.

(9) The activity really enhanced my understanding of the program code covered.

(10) The activity really enhanced my understanding of the concepts and definitions.

Figure 1: The CL self-assessment (based on [33, 49]).

participants who stated that they had no or little previous programming experience. The rest were excluded for a variety of reasons, such as log data suggesting they had "cheated" during the experiment, and missing responses.

Of the 175 participants, 78 self-identified as men and 97 as women, with no-one picking the other options. Most (148) were from the US, fourteen were from India, and the remainder from various countries. English was 158 participants' native language; the rest spoke a mix of languages. Seven of the participants were between 18-24 years of age, 62 were between 25-34, 49 between 35-44, 37 between $45-54$, and 20 were at least 55 years old. 108 of the participants held a bachelor's degree, 21 a master's, one a doctoral degree, and 45 were school graduates. 118 participants reported having some background in the humanities, 41 in natural and technical sciences, and 16 reported no education beyond primary school. 123 had no programming experience at all; 52 reported "a little."

\subsection{Statistical Analyses}

Morrison et al. [49] found a three-factor interpretation for their data, with items targeting IL, EL, and GL forming the three factors, respectively. To replicate that analysis, we grouped the questionnaire items in three, with I1-I3, E1-E3, and G1-G4 forming the factors. We computed Cronbach's alpha to assess the internal reliability of each of these factors separately.

On the other hand, Jiang and Kalyuga [24] found a two-factor interpretation with GL-targeting and IL-targeting items aligned. To replicate that analysis, we grouped the questionnaire items so that E1-E3 again formed one factor and the other seven items (I1-I3 and G1-G4) together formed another.

To investigate whether the three-factor or two-factor model would best fit our data, we conducted two confirmatory factor analyses (CFA) using the two hypothetical models above. Given that our data are ordinal and violate normality assumptions, the DWLS (diagonally weighted least squares) estimator would usually be appropriate. However, Li [35] suggests that with a small sample size $(N<200)$, the ML (maximum likelihood) estimator is more trustworthy.

Following Jiang and Kalyuga [24], we reversed the GL-targeting items' scores for the CFA and item correlations. That is, a score of 10 is treated as a 1 and other scores are similarly converted.

We used the package lavaan in $\mathrm{R}$ (version 4.0).

\section{RESULTS}

\subsection{Reliability}

Cronbach's alpha for all the items was 0.87 , compared to an acceptance threshold of 0.70 . The alpha for the IL-targeting items alone was 0.96; for the EL-targeting items it was 0.89; and for the GL-targeting items it was 0.97 . The IL-targeting and GL-targeting questions together had an alpha of 0.83 . The squared multiple correlations $\left(R^{2}\right)$ of each item varied between 0.61 and 0.95 , thus being above the 0.25 threshold of item reliability.

These results suggest that the instrument and each of the proposed factors is internally reliable.

\subsection{Factor Analyses}

Table 1 shows the fit statistics for the three- and two-factor models.

Table 1: Fit indicators for the hypothesized factor models.

\begin{tabular}{l|l|l}
\hline & $\begin{array}{l}\text { Three Factors } \\
\text { (“IL” / "EL” / "GL”) }\end{array}$ & $\begin{array}{l}\text { Two Factors } \\
\text { (“IL+GL” / “EL”) }\end{array}$ \\
\hline CFI & 0.990 & 0.458 \\
TLI & 0.986 & 0.283 \\
AIC & 5254.0 & 6425.3 \\
BIC & 5358.4 & 6523.4 \\
RMSEA & 0.062 & 0.448 \\
SRMR & 0.033 & 0.320 \\
\hline
\end{tabular}

The Comparative Fit Index (CFI) measures whether the model fits the data better than a more restricted baseline model; the TuckerLewis Index (TLI) is a more conservative estimate of fit. CFI and TLI values above 0.95 are considered a good fit [22]. As Table 1 shows, both CFI and TFI can be considered good for the three-factor model, whereas the two-factor model fits poorly.

The Akaike Information Criterion (AIC) and the related Bayesian Information Criterion (BIC) estimate information loss in the model. In general, lower values of AIC and BIC are better fit estimates. Table 1 shows a lower AIC and BIC for the three-factor model.

RMSEA (The Root Mean Square Error of Approximation) summarizes how closely the model reproduces data patterns. Models with RMSEA values of around 0.07 can be considered a good fit, with lower values preferred. RMSEA confirms the fit of the three-factor model but not the two-factor one.

The Standardized Root Mean Square Residual (SRMR) is defined as the standardized difference between observed and predicted correlations. SRMR values under 0.08 are considered a good fit [22]; again, only the three-factor model meets this criterion. 
Table 2: Factor loadings and descriptive statistics for each questionnaire item.

\begin{tabular}{l|l|l|l|l|l}
\hline Latent Factor & Item & Mean & Std.Err. & Loading & p-value. \\
\hline "Intrinsic" & I1 & 4.03 & 0.122 & 0.887 & $<0.001$ \\
"Intrinsic" & I2 & 4.07 & 0.124 & 0.973 & $<0.001$ \\
"Intrinsic" & I3 & 4.09 & 0.124 & 0.982 & $<0.001$ \\
"Extraneous" & E1 & 1.76 & 0.090 & 0.804 & $<0.001$ \\
"Extraneous" & E2 & 1.71 & 0.082 & 0.896 & $<0.001$ \\
"Extraneous" & E3 & 1.71 & 0.086 & 0.866 & $<0.001$ \\
"Germane" & G1 & $6.65^{*}$ & 0.144 & 0.907 & $<0.001$ \\
"Germane" & G2 & $6.59^{*}$ & 0.135 & 0.967 & $<0.001$ \\
"Germane" & G3 & $6.82^{*}$ & 0.137 & 0.986 & $<0.001$ \\
"Germane" & G4 & $6.75^{*}$ & 0.134 & 0.966 & $<0.001$ \\
\hline
\end{tabular}

\begin{tabular}{|c|c|c|c|c|c|c|c|c|c|}
\hline 11 & $0.8^{* *}$ & $0.87^{* * *}$ & $\begin{array}{l}* * * * \\
0.37\end{array}$ & $\begin{array}{l}* * * \\
0.27\end{array}$ & $\begin{array}{l}* * * * \\
0.30\end{array}$ & 0.48 & 0.047 & 0.11 & 0.06 \\
\hline \&్రా & 12 & 0.94 & $0.4 * *$ & $\begin{array}{l}* * * \\
0.28\end{array}$ & $\begin{array}{c}* * * \\
0.31\end{array}$ & 0.052 & 0.054 & 0.15 & 0.11 \\
\hline $80^{\circ}$ & & & $\begin{array}{l}* * * \\
0.38\end{array}$ & $\begin{array}{l}* * * \\
0.27\end{array}$ & $\begin{array}{l}* * * * \\
0.30\end{array}$ & 0.046 & o.ton & 0.12 & 0.071 \\
\hline & & & & $0.82^{* * *}$ & $0.74^{* * *}$ & $\begin{array}{l}* * * \\
0.49\end{array}$ & $\begin{array}{l}* * * * \\
0.44\end{array}$ & $0.48^{* * *}$ & $\begin{array}{l}* * * \\
0.47\end{array}$ \\
\hline & & & & & $0.79^{* * *}$ & 0.52 & $\begin{array}{l}* * * \\
0.47^{*}\end{array}$ & 0.50 & $\begin{array}{c}* * * \\
0.50\end{array}$ \\
\hline & & & & & & $\begin{array}{l}* * * \\
0.42\end{array}$ & $\begin{array}{l}* * * \\
0.38\end{array}$ & $\begin{array}{l}* * * \\
0.41\end{array}$ & $\begin{array}{l}* * * * \\
0.40\end{array}$ \\
\hline \% & & & & & & 1ी: & $0.89^{* * *}$ & $0 .{ }^{* * *}$ & 0.88 \\
\hline \% & & & & & & ${ }^{\circ} 988^{\circ}$ & $\mathrm{G} 2$ & $0 .{ }^{* * *}$ & 0.93 \\
\hline \% & & & $8_{8} 8^{\circ}$ & & & \% & $\begin{array}{l}88^{\circ} \\
88^{\circ}\end{array}$ & G3 & 0.95 \\
\hline mo & $\theta$ & & & & $\begin{array}{l}88^{8} \\
8^{\circ} \circ\end{array}$ & \% & 承 & \% & G4 \\
\hline
\end{tabular}

Figure 2: Correlations between items, with corresponding scatter-plots. Three asterisks mark significance at $p<0.001$.

Overall, the three-factor model fits our data considerably better than the two-factor model. An ANOVA test confirmed that the three-factor model is a better fit: $\chi^{2}(2)=1175.3, p<0.001$. Table 2 presents the latent factors and factor loadings of the three-factor model. The table also shows the items' means and standard errors.

There are significant, positive correlations between the factors targeting IL and EL $(0.405, p<0.001)$, and between the factors targeting EL and (reversed) GL $(0.447, p<0.001)$, respectively. We found no significant correlation between the IL- and GL-targeting factors $(0.053, p=0.49)$. Figure 2 illustrates the relationships between all the questionnaire items.

Both Figure 2 and Table 2 suggest a floor effect for E1-E3. Note that GL-targeting scores are not reversed.

\section{DISCUSSION AND LIMITATIONS}

\subsection{RQ1: Measuring CL in Programming}

In RQ1, we asked whether Morrison and colleagues' [49] results concerning their CL questionnaire for programming replicate with a different programming tutorial and a different cohort. We found that the questions targeting each CL type aligned with the other questions targeting that type, which supports the claim that the questionnaire has internal reliability. Moreover, we confirm strong support for the questionnaire's discriminant validity-that is, the IL-targeting, EL-targeting, and GL-targeting items appear to be measuring three distinct constructs.

By extension, our findings also strengthen the support for the internal reliability and discriminant validity of Leppink's [33] instrument from which Morrison et al. [49] derived theirs.

\subsection{RQ2: The Two-Factor Model for New CLT}

In RQ2, we asked whether the two-factor model that fit Jiang and Kalyuga's [24] language-learning data well replicates in our programming data. We found a poor fit for the two-factor model that combined IL- and GL-targeting items into a single factor, the other factor consisting of the EL-targeting items. This contrasts with the excellent fit of the three-factor model to our data, with the GL-targeting items in a separate factor. Although the IL- and GL-targeting items aligned in Jiang and Kalyuga's context, they did not in ours.

We feel that the theoretical arguments for New CLT are compelling and that the theory is consistent and parsimonious. However, GL-targeting self-assessment questions do not appear to be redundant with IL across contexts, so their value as empirical evidence for New CLT may be limited. It seems likely that context-dependent and instrument-dependent factors confound the relationship of the IL- and GL-targeting items.

\subsection{Construct Validity in CL Measurement}

Despite the popularity of self-assessments as a way to measure CL types, their construct validity remains largely unproven [32]. There are question marks over whether learners are able to differentiate IL and EL $[44]^{2}$, and the self-assessment of GL is more problematic still. The thread of research to which we add ours has not produced a convincing case for the construct validity of GL-targeting selfassessments. Our study does not resolve this issue either.

What research has shown is that several instruments' GL-targeting items do measure a third construct that is distinct from EL and IL and that this construct replicates in the programming domain. At face value, the third construct (or third constructs; there are differences between instruments [1]) appears to be related to phenomena such as effective learning, motivation, engagement, reflection, efficiency, intensity of cognition, and/or satisfaction with teaching methods.

Many authors (e.g. [29, 33, 34]) have noted that learners can interpret $\mathrm{CL}$ assessments differently from what was intended; subtle changes to wording may be significant. This is a concern in our study as well, and a possible partial explanation for the discrepancy between our findings and those of Jiang and Kalyuga [24], whose questionnaire was similar but not identical to ours [1]. There may also be differences between second-language learning and programming learning-or learners' perceptions of those domains-that contribute to that discrepancy.

\footnotetext{
${ }^{2}$ In the general case, since IL and EL are analytically separated by which aspects of instruction are suboptimal, learners would be required to know instructional design. (And in fact one study did teach learners CLT [29].) Intuition suggests that some forms of EL are much easier for learners to discern from IL than others.
} 


\subsection{Motivation and Cognitive Load}

Motivation and other learner-dependent factors are another caveat of the present study. We did not assess the participants' motivation, and our varied cohort of crowd-sourced learners means that motivations, approaches to learning [41], and self-assessment skills may have varied considerably among the participants.

In 2010, Moreno [47] wrote: "CLT is remarkably silent about the relation among load, affect, and motivation." New CLT sidesteps this critique: it is interested in how instructional design affects "ideal" learners that are highly engaged; it assumes motivation [25, 62]. This is a strength and a weakness. Cordoning off motivation makes New CLT sharper and more robust but also means that the theory must be complemented by other theories and instruments in order to account for real-world situations where students lack motivation. The further research that is needed on the relationship between CLT and motivation has already started outside of CER (e.g., [10, 17, 36])

\subsection{Rapid Changes in Cognitive Load}

An inherent limitation of our study is that we measured CL only after the participants completed a sizeable learning activity. Working memory operates on a timescale of seconds, so many moment-tomoment changes in load are to be expected during a 24-minute video such as ours or the even longer lectures studied by others. Even though subjective post-instruction ratings might provide a general indication of CL levels during instruction, their validity is nonetheless compromised by the extended time frame [25, 26].

Research in CLT has begun to look into "real-time" measurement of CL types [26, 31]; CER should follow suit. In addition to the rapid changes in cognitive load, future research should consider the (comparatively slow but nevertheless fairly frequent) fluctuations in motivation during episodes of learning [13].

\subsection{Summary of Recommendations}

Given our results, researchers in CER and practitioners in programming education may use Morrison's [49] questionnaire with increased confidence in its reliability. That being said, we advise caution in interpreting its GL-targeting scores especially, as their construct validity and theoretical foundation are debatable.

We recommend that researchers in CLT and CER continue to seek empirical evidence for New CLT and instruments to measure CL types. Our study suggests that Jiang and Kalyuga's [24] findings do not necessarily replicate in other contexts. However, even if IL-targeting and GL-targeting subjective assessment items do not combine into a single factor, that does not imply an additive GL as per Old CLT; it merely implies that a third construct is being measured by the instrument.

We agree with authors such as Klepsch et al. [29, 30] that measuring a third, engagement-related construct is meaningful. That construct may not be a discrete source of cognitive load, however. Even if motivation and engagement are external to CLT-as New CLT suggests-research is needed that combines assessments of motivation (e.g. [55]) with measures of cognitive load.

To improve programming education, instructional designs must be evaluated to see how they motivate students and which combinations of CL-driven designs and other activities best facilitate complex learning. CER, as a field, might learn from current research in educational psychology $[10,17,18,27,36]$, which explores the interplay of CLT-based tasks-whose goal is schema constructionwith other learning tasks whose goals are different, such as activating learners' intuitive knowledge, promoting deep approaches to learning, or motivating learning by raising learners' awareness of their knowledge gaps. Productive failure [28] is one example of a framework that might be so used as a complement to CLT (even though there are challenges in reconciling these perspectives [64]).

We join the call $[29,33,34]$ for using a combination of qualitative and quantitative methods to study wording effects in CL questionnaires. We also recommend that CER explore the temporal variation in cognitive load and motivation during learning.

Some of the current CLT-based research in CER cites Old CLT and its constructs seemingly unaware of advances in CLT during the last decade. We hope to raise awareness of some of those advances and encourage the development of ever better cognitive-load measures for programming.

\section{REFERENCES}

[1] Web Appendix. CL questionnaires from [24, 29, 33, 49]. https://osf.io/gk4pe/ ?view only=dbbe398c65544ca5aae94e2ced $73 \mathrm{fc} 71$

[2] Magdalena Andrzejewska and Agnieszka Skawińska. 2020. Examining students' cognitive effort during program comprehension - An eye tracking approach. In International Conference on Artificial Intelligence in Education (AIED '20). 25-30.

[3] Pavlo Antonenko, Fred Paas, Roland Grabner, and Tamara van Gog. 2010. Using electroencephalography to measure cognitive load. Educational Psychology Review 22, 4, 425-438.

[4] Paul Ayres. 2017. Subjective measures of cognitive load: What can they reliably measure? In Cognitive Load Measurement and Application, Robert Z. Zheng (Ed.). Routledge, 9-28.

[5] Esther M. Bergman, Anique B. H. de Bruin, Marc A. T. M. Vorstenbosch, Jan G. M. Kooloos, Ghita C. W. M. Puts, Jimmie Leppink, Albert J. J. A. Scherpbier, and Cees P. M. van der Vleuten. 2015. Effects of learning content in context on knowledge acquisition and recall: A pretest-posttest control group design. BMC Medical Education 15, 133.

[6] Roland Brünken, Susan Steinbacher, Jan L. Plass, and Detlev Leutner. 2002. Assessment of cognitive load in multimedia learning using dual-task methodology. Experimental Psychology 49, 2, 109-119.

[7] Sabine Buchholz and Javier Latorre. 2011. Crowdsourcing preference tests, and how to detect cheating. In Twelfth Annual Conference of the International Speech Communication Association (INTERSPEECH '11).

[8] Michael E. Caspersen. 2007. Educating Novices in the Skills of Programming. Ph.D. Dissertation. Department of Computer Science, University of Aarhus.

[9] Raquel Cerdan, Carmen Candel, and Jimmie Leppink. 2018. Cognitive load and learning in the study of multiple documents. Frontiers in Education 3, 59.

[10] Ouhao Chen and Slava Kalyuga. 2019. Exploring factors influencing the effectiveness of explicit instruction first and problem-solving first approaches. European Journal of Psychology of Education.

[11] Igor Crk and Timothy Kluthe. 2016. Assessing the contribution of the individual alpha frequency (IAF) in an EEG-based study of program comprehension. In 2016 38th Annual International Conference of the IEEE Engineering in Medicine and Biology Society $(E M B C)$. IEEE.

[12] Igor Crk, Timothy Kluthe, and Andreas Stefik. 2016. Understanding programming expertise. ACM Transactions on Computer-Human Interaction 23, 1, 1-29.

[13] Steven C Dang and Kenneth R Koedinger. 2020. The ebb and flow of student engagement: Measuring motivation through temporal pattern of self-regulation. In Proceedings of the 13th International Conference on Educational Data Mining (EDM '20), Anna N. Rafferty, Jacob Whitehill, Cristobal Romero, and Violetta Cavalli-Sforza (Eds.).

[14] Rodrigo Duran, Juha Sorva, and Sofia Leite. 2018. Towards an Analysis of Program Complexity From a Cognitive Perspective. 21-30.

[15] Barbara J. Ericson, Lauren E. Margulieux, and Jochen Rick. 2017. Solving Parsons problems versus fixing and writing code. In Proceedings of the 17th Koli Calling Conference on Computing Education Research (Koli Calling '17). ACM, 20-29.

[16] Pascal W. M. Van Gerven, Fred Paas, Jeroen J. G. Van Merrienboer, and Henk G. Schmidt. 2004. Memory load and the cognitive pupillary response in aging. Psychophysiology 41, 2, 167-174.

[17] Inga Glogger-Frey, Corinna Fleischer, Lisa Grüny, Julian Kappich, and Alexander Renkl. 2015. Inventing a solution and studying a worked solution prepare differently for learning from direct instruction. Learning and Instruction 39, 72-87.

[18] Inga Glogger-Frey, Katharina Gaus, and Alexander Renkl. 2016. Learning from direct instruction: Best prepared by several self-regulated or guided invention activities? Learning and Instruction 51, 26-35. 
[19] Oiang Hao, David H. Smith IV, Naitra Iriumi, Michail Tsikerdekis, and Amy J. Ko. 2019. A systematic investigation of replications in computing education research. ACM Transactions on Computing Education 4, 42.

[20] Kyle J Harms, Jason Chen, and Caitlin Kelleher. 2016. Distractors in Parsons problems decrease learning efficiency for young novice programmers. In The 12th International Computing Education Research Conference (ICER '16). ACM 241-250.

[21] Kenneth Holmqvist, Marcus Nyström, Richard Andersson, Richard Dewhurst, Jarodzka Halszka, and Joost van de Weijer. 2011. Eye tracking: A comprehensive guide to methods and measures. Oxford University Press, Oxford New York.

[22] Li-Tze Hu and Peter M. Bentler. 1999. Cutoff criteria for fit indexes in covariance structure analysis: Conventional criteria versus new alternatives. Structural Equation Modeling: A Multidisciplinary fournal 6, 1, 1-55.

[23] Panagiotis G Ipeirotis, Foster Provost, and Jing Wang. 2010. Quality management on Amazon Mechanical Turk. In Proceedings of the ACM SIGKDD workshop on human computation. 64-67.

[24] Dayu Jiang and Slava Kalyuga. 2020. Confirmatory factor analysis of cognitive load ratings supports a two-factor model. 16, 3, 216-225.

[25] Slava Kalyuga. 2011. Cognitive load theory: How many types of load does it really need? Educational Psychology Review 23, 1-19.

[26] Slava Kalyuga and Jan L Plass. 2017. Cognitive load as a local characteristic of cognitive processes: Implications for measurement approaches. In Cognitive Load Measurement and Application, Robert Z. Zheng (Ed.). Routledge, 59-74.

[27] Slava Kalyuga and Anne-Marie Singh. 2016. Rethinking the boundaries of cognitive load theory in complex learning. Educational Psychology Review 28, 831-852.

[28] Manu Kapur. 2008. Productive failure. Cognition and Instruction 26, 3, 379-424.

[29] Melina Klepsch, Florian Schmitz, and Tina Seufert. 2017. Development and validation of two instruments measuring intrinsic, extraneous, and germane cognitive load. Frontiers in Psychology 8.

[30] Melina Klepsch and Tina Seufert. 2020. Understanding instructional design effects by differentiated measurement of intrinsic, extraneous, and germane cognitive load. Number 48. Springer Netherlands. 45-77 pages.

[31] Andreas Korbach, Roland Brünken, and Babette Park. 2018. Differentiating different types of cognitive load: A comparison of different measures. Educational Psychology Review 30, 503-529.

[32] Jimmie Leppink. 2020. Revisiting cognitive load theory: Second thoughts and unaddressed questions. Scientia Medica $30,1-8$

[33] Jimmie Leppink, Fred Paas, Cees P. M. Van der Vleuten, Tamara Van Gog, and Jeroen J. G. Van Merriënboer. 2013. Development of an instrument for measuring different types of cognitive load. Behavior research methods 45, 1058-72.

[34] Jimmie Leppink, Fred Paas, Tamara van Gog, Cees P. M. van der Vleuten, and Jeroen J. G. van Merriënboer. 2014. Effects of pairs of problems and examples on task performance and different types of cognitive load. Learning and Instruction $30,32-42$.

[35] Cheng-Hsien Li. 2016. Confirmatory factor analysis with ordinal data: Comparing robust maximum likelihood and diagonally weighted least squares. Behavior research methods 48, 3, 936-949.

[36] Vicki Likourezos and Slava Kalyuga. 2017. Instruction-first and problem-solving first approaches: alternative pathways to learning complex tasks. Instructional Science 45, 2, 195-219.

[37] Lauri Malmi, Ahmad Taherkhani, Judy Sheard, Roman Bednarik, Juha Helminen, Päivi Kinnunen, Ari Korhonen, Niko Myller, and Juha Sorva. 2014. Theoretical underpinnings of computing education research: What is the evidence?. In Proceedings of the Tenth Annual Conference on International Computing Education Research (ICER '14). ACM, New York, New York, USA, 27-34.

[38] Lauren Margulieux and Richard Catrambone. 2017. Using learners' selfexplanations of subgoals to guide initial problem solving in App Inventor. In The 2017 ACM Conference on International Computing Education Research. 21-29.

[39] Lauren Margulieux, Tuba Ayer Ketenci, and Adrienne Decker. 2019. Review of measurements used in computing education research and suggestions for increasing standardization. Computer Science Education.

[40] Lauren E. Margulieux, Briana B. Morrison, and Adrienne Decker. 2020. Reducing withdrawal and failure rates in introductory programming with subgoal labeled worked examples. International fournal of STEM Education 7, 1.

[41] Ference Marton and Roger Säljö. 1976. On qualitative differences in learning: I Outcome and process. British fournal of Educational Psychology 46, 1, 4-11

[42] Raina Mason and Graham Cooper. 2012. Why the bottom $10 \%$ just can't do it Mental effort measures and implication for introductory programming courses In Proceedings of the 14th Australasian Conference on Computing Education (ACE 12) (CRPIT, Vol. 123), Michel de Raadt and Angela Carbone (Eds.). Australian Computer Society, 187-196.

[43] Raina Mason, Graham Cooper, and Michael Raadt. 2012. Trends in introductory programming courses in Australian Universities - Languages, environments and pedagogy. Conferences in Research and Practice in Information Technology Series 123, 33-42.

[44] Myrto F. Mavilidi and Lijia Zhong. 2019. Exploring the development and research focus of cognitive load theory, as described by its founders: Interviewing John Sweller, Fred Paas, and Jeroen van Merriënboer. Educational Psychology Review
31, 499-508.

[45] Ritayan Mitra, Karen S. McNeal, and Howard D. Bondell. 2016. Pupillary response to complex interdependent tasks: A cognitive-load theory perspective. Behavior Research Methods 49, 5, 1905-1919.

[46] Jan Moons and Carlos De Backer. 2013. The design and pilot evaluation of an interactive learning environment for introductory programming influenced by cognitive load theory and constructivism. Computers \& Education 60, 1, 368-384.

[47] Roxana Moreno. 2010. Cognitive load theory: More food for thought. Instructional Science 38, 2, 135-141.

[48] Briana B. Morrison. 2017. Dual modality code explanations for novices: Unexpected results. In The 2017 ACM Conference on International Computing Education Research (ICER '17). ACM, 226-235.

[49] Briana B. Morrison, Brian Dorn, and Mark Guzdial. 2014. Measuring cognitive load in introductory CS: Adaptation of an instrument. In Proceedings of the Tenth Annual Conference on International Computing Education Research (ICER '14). ACM, 131-138.

[50] Briana B. Morrison, Lauren E. Margulieux, and Mark Guzdial. 2015. Subgoals, context, and worked examples in learning computing problem solving. Proceedings of the eleventh annual International Conference on International Computing Education Research - ICER '15, 21-29.

[51] Nargess Nourbakhsh, Yang Wang, and Fang Chen. 2013. GSR and blink features for cognitive load classification. In IFIP Conference on Human-Computer Interaction (INTERACT '13). Springer, 159-166.

[52] Nargess Nourbakhsh, Yang Wang, Fang Chen, and Rafael A. Calvo. 2012. Using galvanic skin response for cognitive load measurement in arithmetic and reading tasks. In Proceedings of the 24th Australian Conference on Computer-Human Interaction ( $\mathrm{OzCHI}$ '12). ACM.

[53] Fred G. Paas. 1992. Training strategies for attaining transfer of problem-solving skill in statistics: A cognitive-load approach. Journal of Educational Psychology 84, 4, 429-434.

[54] Fred G. W. C. Paas, Jeroen J. G. van Merriënboer, and Jos J. Adam. 1994. Measurement of cognitive load in instructional research. Perceptual and Motor Skills 79, 1, $419-430$

[55] Günter Daniel Rey and Florian Buchwald. 2011. The expertise reversal effect: Cognitive load and motivational explanations. fournal of Experimental Psychology: Applied 17, 1, 33-48.

[56] Anthony V. Robins, Lauren E. Margulieux, and Briana B. Morrison. 2019. Cognitive sciences for computing education. In The Cambridge Handbook of Computing Education Research, Sally A. Fincher and Anthony V. Robins (Eds.). Cambridge University Press, 231-275.

[57] Wolfgang Schnotz and Christian Kürschner. 2007. A reconsideration of cognitive load theory. Educational Psychology Review 19, 469-508.

[58] Yu Shi, Natalie Ruiz, Ronnie Taib, Eric Choi, and Fang Chen. 2007. Galvanic skin response (GSR) as an index of cognitive load. In Extended Abstracts Proceedings of the 2007 Conference on Human Factors in Computing Systems (CHI '07). ACM Press.

[59] Teemu Sirkiä and Juha Sorva. 2015. Tailoring animations of example programs. In Proceedings of the 15th Koli Calling Conference on Computing Education Research (Koli Calling '15). ACM, 147-151.

[60] Ben Skudder and Andrew Luxton-Reilly. 2014. Worked examples in computer science. In Proceedings of the 16th Australasian Conference on Computing Education (ACE '14) (CRPIT, Vol. 148), Jacqueline Whalley and Daryl D'Souza (Eds.). Australian Computer Society, 59-64.

[61] Juha Sorva and Otto Seppälä. 2014. Research-based design of the first weeks of CS1. In Proceedings of the 14th Koli Calling International Conference on Computing Education Research (Koli Calling '14). ACM, 71-80.

[62] John Sweller. 2010. Element interactivity and intrinsic, extraneous, and germane cognitive load. Educational Psychology Review 22, 123-138.

[63] John Sweller, Jeroen J G Merriënboer, and Fred Paas. 2019. Cognitive architecture and instructional design: 20 years later. Educational Psychology Review.

[64] John Sweller and Fred Paas. 2017. Should self-regulated learning be integrated with cognitive load theory? A commentary. Learning and Instruction 51, 85-89.

[65] John Sweller, Jeroen J. G. van Merriënboer, and Fred G. W. C. Paas. 1998. Cognitive architecture and instructional design. Educational Psychology Review 10, 251-296.

[66] Jeroen J. G. van Merriënboer. 1990. Strategies for programming instruction in high school: Program completion vs. program generation. Fournal of Educational Computing Research 6, 3, 265-285.

[67] Robert R. Whelan. 2007. Neuroimaging of cognitive load in instructional multimedia. Educational Research Review 2, 1, 1-12.

[68] Alexander Seeshing Yeung, Cynthia Fong King Lee, Isabel Maria Pena, and Jeni Ryde. 2000. Toward a subjective mental workload measure. In Paper presented at the International Congress for School Effectiveness and Improvement.

[69] Robert Z. Zheng (Ed.). 2017. Cognitive Load Measurement and Application. Routledge.

[70] Rui Zhi, Thomas W Price, Samiha Marwan, Alexandra Milliken, Tiffany Barnes, and Min Chi. 2019. Exploring the impact of worked examples in a novice programming environment. In Proceedings of the 50th ACM Technical Symposium on Computer Science Education (SIGCSE '19). ACM, 98-104. 